\title{
Study of the Application of Noise Immunity in Radio Communication Systems for Special Courses
}

\author{
Tulyaganov Abdukhalil, Nozima Yadgarova
}

Electronics and Radio Engineering Department, Tashkent University of Information Technologies Named After Muhammad Al-Khwarizmi, Tashkent, Uzbekistan

Email address:

a.tulyaganov@tuit.uz(T.Abdukhalil),nozima_yadgarova@mail.ru (N. Yadgarova)

\section{To cite this article:}

Tulyaganov Abdukhalil, Nozima Yadgarova. Study of the Application of Noise Immunity in Radio Communication Systems for Special Courses. Bioprocess Engineering. Vol. 2, No. 2, 2018, pp. 20-23. doi: 10.11648/j.be.20180202.11

Received: March 1, 2018; Accepted: March 21, 2018; Published: April 18, 2018

\begin{abstract}
The interference immunity of radio communication systems has always been considered an actual topic. Especially when it comes to information in military. In the modern theory of military confrontation, more and more importance is attached to the introduction of new control systems based on network-centric principles, while the basis of such a control system is the communication subsystem. In this connection, on the one hand, communication systems must meet the stringent requirements of the system of command and control of troops and weapons, on the other hand, in the conditions of modern confrontation, they themselves are subjected to destructive influence from the enemy. The basis for modern destructive impact on radio communication systems (RCS) are the use of electronic warfare (EW) and electronic suppression (ES). The methodology for assessing the impact of the ES on the RCS is now sufficiently developed, which updates the research directions in the field of interference protection of the RCS. This article is aimed at the prepared reader and is addressed to teachers who teach at military universities and classifies about the research carried out to improve noise immunity of the RCS in the dynamics of the EW. A comparative analysis of methods for increasing the noise immunity of the RCS is presented. It is reasonable to consider the method of pseudo-random adjustment of the operating frequency of the hopper as one of the fundamental in solving problems of increasing noise immunity. The main types of interference affecting the RCS and their classification are considered. The notion of electronic warfare as a basis for modeling the processes of interaction between the RCS and the EW (S) means is introduced. The main methodological approaches to the modeling of electronic conflict, the features of using a different mathematical apparatus for such modeling are considered. The indicators of stealth and noise immunity of the RCS in the application of the pseudo-random tuning of the operating frequency regime in the dynamics of the electronic conflict are presented.
\end{abstract}

Keywords: Electronic Suppression, Electronic Warfare, Radio Communication Systems

\section{Introduction}

The purpose of passive radio masking is to reduce the signal energy available to the reconnaissance vehicle in order to reduce its visibility. There are at least several ways for this. First, you need to design protected systems so that they work with the possible lower levels of radiation. Such an effect is achieved due to the correct choice of signal structures and properties, as well as acceptable ways of signal processing in receivers. Secondly, it is necessary to use broadband signals (signals with a large base B $=$ DfT $>>1$ ). Such signals may be useful to the receiver of reconnaissance means. Stealth operation of transmitters depending on the frequency range in the range up to several tens of MHz. Transmitters with IF modulation or dual modulation can be used. Thirdly, it is necessary to reduce the levels of spurious and unintentional emissions of radio electronic systems and means of reconnaissance objects. For basic radiations, there should be a proxy interchange with the receivers of reconnaissance means.

The first two methods, of course, refer to the provision of systems that are protected from intelligence, but go beyond the actual conflict between two RE. Another thing is special ways to reduce the levels of unintended, incidental and secondary (reflected) emissions.

Side beams are created by any radio and telecommunication systems due to emissions on the 
harmonics and subharmonics of the carrier, at the combination frequencies of various oscillations used to form the main radiated signal. In radio transmitters of spurious radiation, antennas (radiation along the side lobes of the radiation patterns) and a feeder path are also created. It is characteristic that all this knowledge lies outside the main spatial-frequency band of radiation masked from reconnaissance signals.

Unintentional discharges are created by parasitic oscillations that arise in electronic circuits and fall on the main band of the useful signal spectrum, but when they do not emit radiation. For example, in the control and testings, installation and adjustment, routine maintenance on the device, including radio frequency systems.

Secondary (reflected) excretions are created when active influence on the reconnaissance means by signals of different frequency and shape. This is reflected radar from the objects of reconnaissance. Secondary also includes reradiation of radioelectronic means, modulated by an information signal, with their accidental and / or deliberate irradiation with highfrequency signals (high-frequency imposition).

Basic technical means preventing collateral, unintentional and secondary emissions while protecting RES from the radio and radio technical intelligence services provide for: suppression of parasitic generations - sources of spurious emissions, shielding of equipment from external electromagnetic fields and to weaken their own radiations and filter interference.

Suppression of the source of interference is carried out by the optimal design of electrical circuits and the layout of printed circuit boards, taking into account the requirements for minimizing spurious generation, created internal elements of devices and circuitry. These measures include the following objectives: isolation of power supply circuits, elimination of radiating conductors, reconstruction or elimination of especially noisy (generating) circuits.

Screening is a constructive normal attenuation of any radiation and is of great importance both from the point of view of compliance with requirements and to prevent the emission of information signals at base frequencies and through parasitic generations.

Filtration is the main and remote suppression (attenuation) of interference in power and ground circuits, in signal circuits. Interference suppression filters can reduce interference from both external and internal sources of interference.

To mask from the radar reconnaissance means, it is necessary to reduce the level of the reflected signal or, what is the same, to reduce the effective scattering surface of the target (ESS). The latest achievement is due to the use of special non-reflective forms of reconnaissance objects, and through the use of measures to increase the energy absorbed by the targets (through the use of special coatings and other methods and means).

\section{Main Part}

As noted above, traditional methods of radio masking, based on shielding, regulation of radiation work, etc. do not always solve all the problems of concealing radio electronic systems and means from radio electronic reconnaissance. Sufficient indicators of the invisibility of the RES are not provided by the choice of the geographic location of the object: in any case, concealed means are available for exploration from space, and often for reconnaissance by means of portable technical means. In such cases, as an alternative to passive types of camouflage, and as a possible addition, active radio technical camouflage (ARTC) is used.

The term ARTC, as it is understood at present, means countering radio and radio technical intelligence by creating special interference fields that hamper unauthorized reception of a signal by means of electronic intelligence and the allocation of messages by means of radio reconnaissance. The definition is incomplete without an essential limitation: interference with active radio cloaking should not interfere with the operation of masked systems, those, they should not reduce their performance and quality indicators below some acceptable level. Active interference to radio intercept equipment is generated by transmitters installed on the ground or on aircraft and ships. In order to protect the cable communication lines and objects, electromagnetic noise is applied to the surrounding space in the required frequency range using special noise generators.

The radar operating in the survey mode generates continuous active noise and impulse noise. The effect of active noise interference is the masking of useful signals in a certain solid angle and a certain range of ranges. As a result, the radar detection characteristics, their resolving power and the accuracy of determining the coordinates and parameters of the target movement are significantly deteriorating. The sources of passive interference for radars can be radar traps and false targets (for example, ejected metal strips) that mimic or mask targets (radio-contrast objects).

According to the nature of the impact on radio electronic system, they distinguish between masking and imitating passive and active interference. Masking interference makes it difficult to detect and recognize a useful signal. Simulated interference is intended to introduce false information into the suppressed RES. These disturbances are a fake for a useful signal. Sometimes imitating interference carries an overload of reconnaissance information channels of RES, as a result of which the hardware capacity of reconnaissance receivers decreases, and some useful information is lost.

The task of electronic countermeasures, in the case of active jamming, is to increase the error rate during the identification of the protected signal by the reconnaissance receiver. To exclude the possibility of filtering, the simulating interference signal differs slightly from the useful one by unimportant (uninformative) simulated parameters. At the same time, the information parameter is distorted significantly. By information here is meant a parameter that is measured in a suppressed RES.

Depending on the width of the spectrum, there are noise barriers and sighting. The width of the spectrum of the blocking noise is many times higher than the bandwidth of 
the receiver of the suppressed RES. The aiming interference has a spectrum, the width of which is consistent with the bandwidth of the receiver. The spectral density of the aiming noise is greater than the interference of the barrier. However, the use of impact interference requires a very accurate reconnaissance of the carrier frequencies of the RES.

Masking and simulating interference can be additive and multiplicative (modulating). The first prevent the correct reception of useful signals due to the addition of the interference signal to the useful signal. Multiplicative interference parasitic modulation of the useful signal and thereby distort its parameters (amplitude, phase, frequency, polarization).

Noise interference is generated by random and pseudorandom signal (number) generators.

Random signals include signals that can not be determined at any time, pseudorandom signals include signals that are close in their characteristics to random ones, but are periodic, instantaneous values of which at any time in principle, but can be determined. As a source of the random process, for the formation of noise signals, for example, electron fluctuations in noise diodes can be used, in the pseudorandom signal generators, the primary source of the random process is absent, and the signal is generated according to a predetermined algorithm.

\section{$\mathrm{U}$}

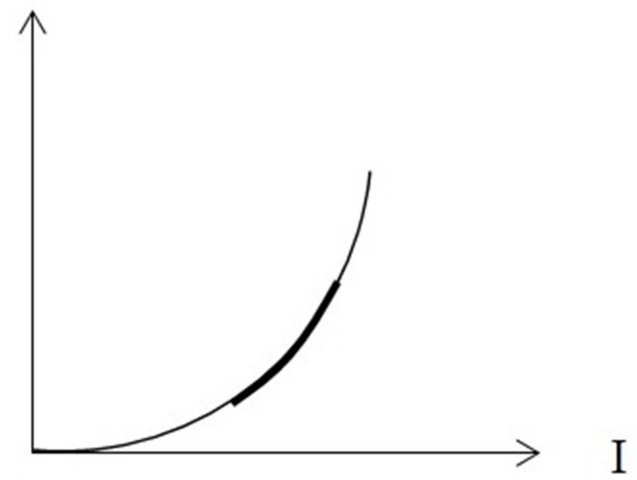

Figure 1. Volt-ampere characteristic of a noise diode. Darkly distinguished area of fluctuations.

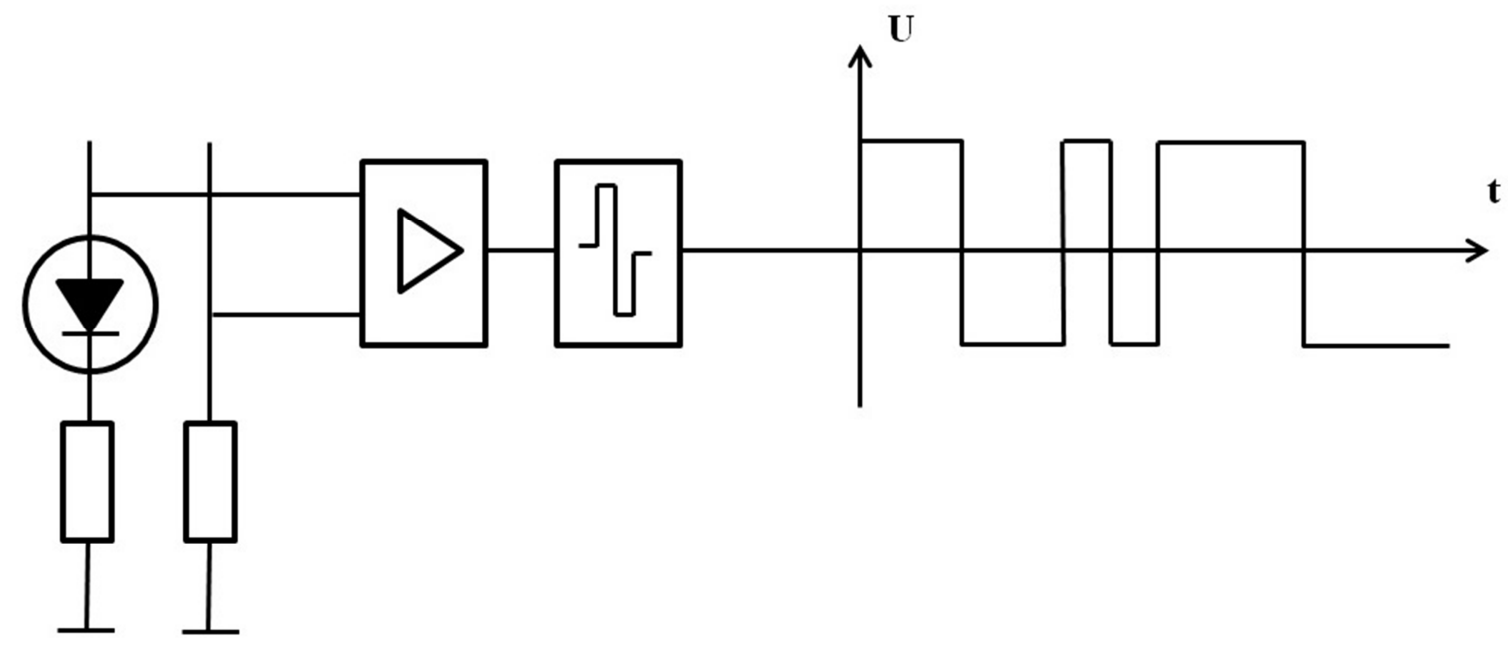

Figure 2. Simplified scheme of the random signal generator.

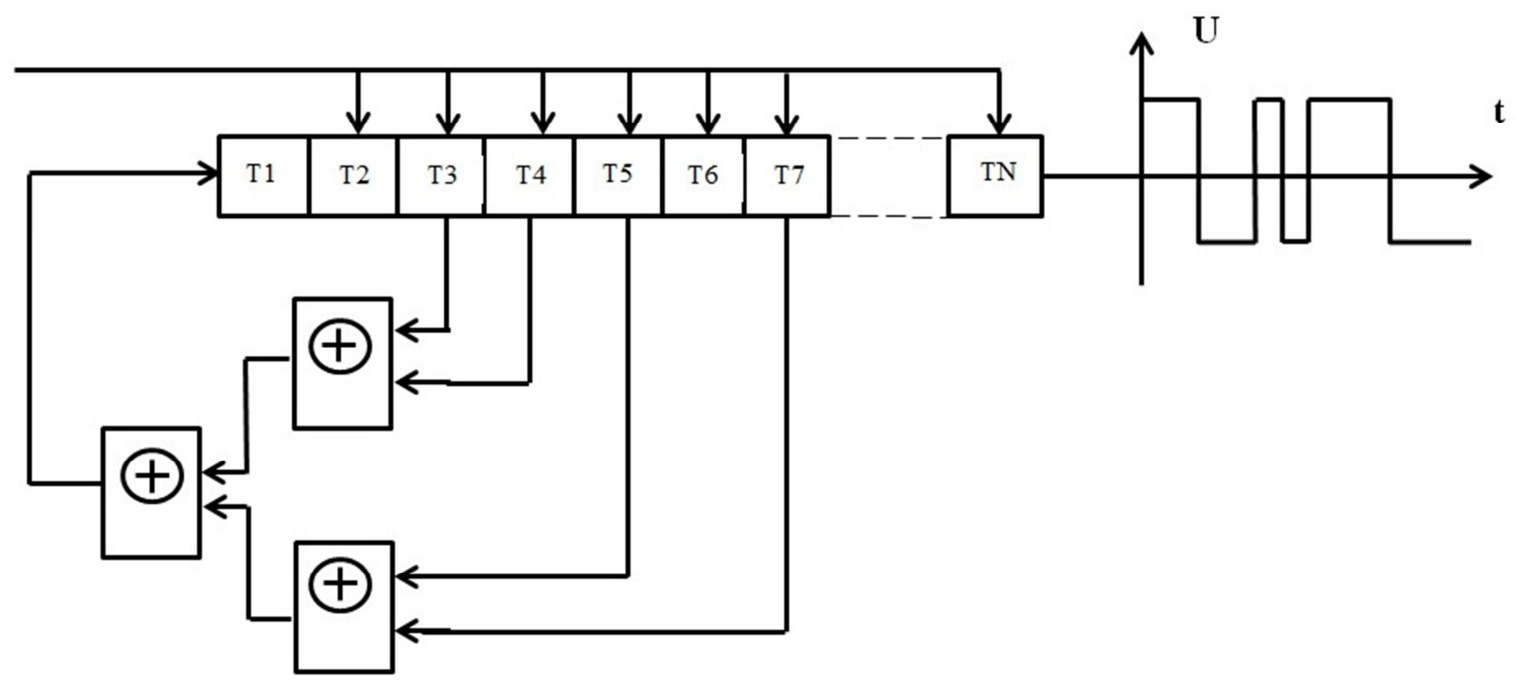

Figure 3. Simplified scheme of the pseudorandom signal generator. 
Despite the main advantage of random signals, unpredictability, the required spectral characteristics of noise in random signal generators are quite difficult to realize, but a well-developed theory of pseudorandom signals allows the construction of relatively simple pseudorandom noise generators. Therefore, many well-known and widely used methods of producing a "noise" signal in fact form a pseudonoise signal, for a number of its frequency and time parameters very close to the actual noise (with an unlimited growth of the period of the pseudorandom signal, its characteristics tend to a random signal close to it in structure) but in fact largely deterministic or having significant internal correlation links. In many cases, this signal can completely replace the noise signal (when measuring frequency characteristics, estimating noise immunity, etc.). In fact, the signal's determinism is even useful, since it facilitates its parameterization and stabilization. A signal having internal correlation links can be successfully used and as a protective noise if interception is conducted without using correlative processing of the received or pre-recorded mixture of the useful signal-to-noise ratio. However, when applying relatively simple methods of correlation processing, such a "noise" can almost always be completely suppressed.

Use of simulated interference:

In some cases, imitating interference is used to disorient radar systems, which create false marks on the radar screen.

Imitating active interference is created by the response stations. Active imitating interference is divided into single and multiple.

A single response is a radio pulse emitted in response to a received signal from suppressed radar with some delay. The delay time varies so as to create on the radar screen an imitation of a really moving target. At a sufficiently high power of the interference transmitter due to the action through the side lobes of the radiation patterns of the antenna, several false marks are created on the radar screen moving at a certain speed, which considerably complicates the detection work.

Multiple response interference is a series of radio pulses emitted in response to a received signal from suppressed radar. Radio impulses of interference and useful reflected signal in form, duration and power are identical.

\section{Conclusion}

In conclusion let's talk about interference stations, including the reconnaissance receiver and the interference transmitter, is characterized by the maximum and minimum range of action. The maximum distance of the interference station is the maximum distance between the suppressed radar and the interference station at which the reconnaissance device of the interference station detects and identifies the radar with a given probability of correct detection and false alarm. The value of the maximum range is determined mainly by the parameters of the radar, the sensitivity of the reconnaissance receiver and the conditions of line of sight. For transmitters of interference that do not have receiving reconnaissance devices, the maximum distance is understood as the distance between the radar and the interference transmitter in which the interference power at the input of the suppressed radar receiver achieves its real sensitivity.

\section{References}

[1] Tsvetnov V. V. (1999). Electronic warfare: radio masking and noise protection: a tutorial. Moscow.

[2] Maksimov M. V. (1976). Protection against radio interference. M.: Soviet radio.

[3] Nikolenko N. F. (1987). Fundamentals of the theory of electronic warfare. M.: Military publishing house.

[4] Shirman Y. D. (2007). Radioelectronic systems of the basis of construction and theory: reference book. M: Radio Engineering, 2 edition.

[5] Kupriyanov A. I. (2011). Electronic warfare. Fundamentals of the theory. Moscow: The University Book.

[6] Perunov Y. M. (2008). Radio-electronic suppression of information channels and weapon control systems. M.: Radio engineering.

[7] Kondratenko S.V. (2018). Analysis of noise immunity at common circuits of the front end parts of high- speed transceivers. IOP Publishing.

[8] Elena Fimmel, Lutz Strüngmann. (2018). Mathematical fundamentals for the noise immunity of the genetic code. Bio Systems.

[9] Vyboldin Yu. K., Borisov S.V. (2018). Interference effect of impulse noise on noise immunity of communication and control channels in underground structures. IOP Publishing.

[10] Licklider J. C. R., Goffard S. J. (2018). Effects of Noise on Radio Communication. Acoustical society of America. 